\title{
NUMBER OF ODD BINOMIAL COEFFICIENTS
}

\section{HEIKO HARBORTH}

\begin{abstract}
Let $F(n)$ denote the number of odd numbers in the first $n$ rows of Pascal's triangle, and $\theta=(\log 3) /(\log 2)$. Then $\alpha=\lim \sup F(n) / n^{\theta}=$ 1 , and $\beta=\lim \inf F(n) / n^{\theta}=0.812556 \ldots$
\end{abstract}

It is known that almost all binomial coefficients are even numbers (see for example [1]-[3]). This means

$$
\lim _{n \rightarrow \infty} F(n) /\left(\begin{array}{c}
n+1 \\
2
\end{array}\right)=\lim _{n \rightarrow \infty} F(n) / n^{2}=0
$$

if $F(n)$ denotes the number of odd numbers in the first $n$ rows of Pascal's triangle. Recently in [4] and [5] it is asked more precisely for the asympiotic behavior of $F(n)$. Let

$$
\alpha=\lim _{n \rightarrow \infty} \sup F(n) / n^{\theta}, \quad \beta=\lim _{n \rightarrow \infty} \inf F(n) / n^{\theta},
$$

and

$$
\theta=(\log 3) /(\log 2)=1.584962 \ldots
$$

Then it is shown in [5] that

$$
1 \leqslant \alpha \leqslant 1.052 \text {, and } 0.72 \leqslant \beta \leqslant(9 / 7)(3 / 4)^{\theta} \leqslant 0.815 \text {. }
$$

Furthermore it is conjectured that 1 and $(9 / 7)(3 / 4)^{\theta}=3^{\theta} / 7=0.814931 \ldots$ are the true values of $\alpha$ and $\beta$. In this note we will prove $\alpha=1$ and $\beta=0.812556 \ldots$

THEOREM 1. $\alpha=1$.

Proof. Since

$$
\left(\begin{array}{l}
n \\
0
\end{array}\right)=\left(\begin{array}{l}
n \\
n
\end{array}\right)=1, \quad \text { and }\left(\begin{array}{c}
n \\
i
\end{array}\right) \equiv 0 \quad(\bmod 2), \quad 1 \leqslant i \leqslant n-1,
$$

for $n=2^{r}, r=0,1, \ldots$, we have the recursion

$$
F\left(2^{r}+x\right)=F\left(2^{r}\right)+2 F(x), \quad 0 \leqslant x \leqslant 2^{r}, \quad r=0,1, \ldots,
$$

if, in addition, $F(0)=0$ is defined. From (3), by induction on $r$, we get

$$
F\left(2^{r}\right)=3^{r}
$$

and thus $F\left(2^{r}\right) / 2^{r \theta}=3^{r} / 2^{r \theta}=1$ for all $r$, which yiclds $\alpha \geqslant 1$.

Next we assert

Received by the editors March 19, 1976.

AMS (MOS) subject classifications (1970). Primary 10L10, 10A30.

(C) American Mathematical Society 1977 
(5)

$$
F\left(2^{r}+x\right) /\left(2^{r}+x\right)^{\theta} \leqslant 1 \text { for } 0 \leqslant x \leqslant 2^{r}, \quad r=0,1, \ldots
$$

This is true for $r=0$. If we assume the validity of (5) for all natural numbers $\leqslant r-1$, we can use $F(x) \leqslant x^{\theta}$ for $0 \leqslant x \leqslant 2^{r}$ to get from (3) and (4) that

$$
\frac{F\left(2^{r}+x\right)}{\left(2^{r}+x\right)^{\theta}}=\frac{F\left(2^{r}\right)+2 F(x)}{\left(2^{r}+x\right)^{\theta}} \leqslant \frac{3^{r}+2 x^{\theta}}{\left(2^{r}+x\right)^{\theta}}=f(x), \quad 0 \leqslant x \leqslant 2^{r} .
$$

From

$$
\frac{d f}{d x}=\frac{\theta}{\left(2^{r}+x\right)^{\theta+1}}\left(2^{r+1} x^{\theta-1}-3^{r}\right)=0
$$

it follows that $f(x)$ has exactly one extremum. This together with $f(0)=f\left(2^{r}\right)$ $=1$ and $f\left(2^{r-1}\right)=5 / 3^{\theta}<1$ yields $f(x) \leqslant 1$ for $0 \leqslant x \leqslant 2^{r}$. Thus (5) is proved by induction on $r$, and from (5) we conclude $\alpha \leqslant 1$.

THEOREM 2. $\beta=0.812556 \ldots$

Proof. We consider the sequence

$$
\left\{q_{r}\right\}=\left\{F\left(n_{r}\right) / n_{r}^{\theta}\right\} \text { with } n_{r}=2 n_{r-1} \pm 1, \quad n_{0}=1,
$$

where + or - is chosen so that $q_{r}$ becomes minimal. So for $r=1,2, \ldots, 25$ we have to choose

(7) +-+-++-+-++-+-+-++-+-++-+ .

If $t_{r}$ denotes the sum of the binary digits of $n_{r}$, the first eleven values of $n_{r}$, $F\left(n_{r}\right)$, and $t_{r}$ are

$\begin{array}{rrrr}r & n_{r} & F\left(n_{r}\right) & t_{r} \\ 0 & 1 & 1 & 1 \\ 1 & 3 & 5 & 2 \\ 2 & 5 & 11 & 2 \\ 3 & 11 & 37 & 3 \\ 4 & 21 & 103 & 3 \\ 5 & 43 & 317 & 4 \\ 6 & 87 & 967 & 5 \\ 7 & 173 & 2869 & 5 \\ 8 & 347 & 8639 & 6 \\ 9 & 693 & 25853 & 6 \\ 10 & 1387 & 77623 & 7\end{array}$

LEMMA. $\left\{q_{r}\right\}$ is strictly decreasing.

Proof. We suppose

(8) $F\left(2 n_{r}+1\right) /\left(2 n_{r}+1\right)^{\theta} \geqslant q_{r}$ and $F\left(2 n_{r}-1\right) /\left(2 n_{r}-1\right)^{\theta} \geqslant q_{r}$.

Using (3), (4), and the binary representation of $n_{r}$ we obtain

$$
F\left(2 n_{r} \pm 1\right)=3 F\left(n_{r}\right) \pm 2^{t_{r}}, \quad t_{r}=t_{r-1}+\frac{1}{2} \pm \frac{1}{2} .
$$


(Here the reader may recognize the well-known result (see [5] for references) that the number of odd $\left(\begin{array}{c}n \\ i\end{array}\right)$ is $2^{t}$, where $t$ is the number of binary digits of $n$.) We insert (9) and (6) in (8), and substitute $2 n_{r}=a$ and $2^{t_{r}} /\left(3 F\left(n_{r}\right)\right)=b$ to get

$$
\begin{aligned}
1+b \geqslant & \left(1+\frac{1}{a}\right)^{\theta}=1+\frac{\theta}{a}+\frac{\theta(\theta-1)}{2 a^{2}} \\
& +\theta(\theta-1) \sum_{i=1}^{\infty}\left(\frac{-1}{a}\right)^{i+2} \frac{(2-\theta) \cdots(i+1-\theta)}{(i+2) !}, \\
1-b \geqslant & \left(1-\frac{1}{a}\right)^{\theta}=1-\frac{\theta}{a}+\frac{\theta(\theta-1)}{2 a^{2}} \\
& +\theta(\theta-1) \sum_{i=1}^{\infty}\left(\frac{1}{a}\right)^{i+2} \frac{(2-\theta) \cdots(i+1-\theta)}{(i+2) !}
\end{aligned}
$$

Addition of the last two inequalities yields the contradiction

$$
2 \geqslant 2+\theta(\theta-1) / a^{2}+\cdots>2 \text {. }
$$

Thus the inequalities (8) cannot both be true, which proves the Lemma.

Now $q_{r}>0$ together with the Lemma proves the convergence of $\left\{q_{r}\right\}$. It follows that

$$
B \leqslant q=\lim _{r \rightarrow \infty} q_{r}<q_{19}=0.812556 \ldots
$$

with

$$
\begin{aligned}
n_{19} & =710317 \\
& =2^{19}+2^{17}+2^{15}+2^{14}+2^{12}+2^{10}+2^{9}+2^{7}+2^{5}+2^{3}+2^{2}+1 .
\end{aligned}
$$

We still have to prove

$$
F(n) / n^{\theta}>0.812556=\gamma .
$$

This is true for $1 \leqslant n \leqslant 2$, and we assume the validity of (11) for $1 \leqslant n \leqslant 2^{r}$. To obtain the step from $r$ to $r+1$ in a proof of (11) by induction on $r$ we have to conclude from this assumption that (11) also holds for $n=2^{r}+x$, $1 \leqslant x \leqslant 2^{r}$. We divide this interval into eleven intervals:

$$
\begin{aligned}
n & =2^{r-s} m+x, \quad 1 \leqslant x \leqslant 2^{r-s}, \\
m & =n_{s} \text { for } s=1,3,6,8,10, \\
m & =n_{s}-1 \text { for } s=2,4,5,7,9,10 .
\end{aligned}
$$

Let $t$ be the sum of the binary digits of $m$, and $2^{s}<m<2^{s+1}$. Then for $1 \leqslant x \leqslant 2^{r-s}$ we get from (3) and (4) that

$$
\frac{F\left(2^{r-s} m+x\right)}{\left(2^{r-s} m+x\right)^{\theta}}=\frac{3^{r-s} F(m)+2^{t} F(x)}{\left(2^{r-s} m+x\right)^{\theta}}>\frac{3^{r-s} F(m)+2^{t} \gamma x^{\theta}}{\left(2^{r-s} m+x\right)^{\theta}}=f_{s}(x) \text {. }
$$

The unique extremum of $f_{s}(x)$ is a minimum at

$$
x_{\min }=2^{r-s}\left(F(m) / \gamma m 2^{t}\right)^{1 /(\theta-1)} \text {. }
$$


For $m=n_{s}$ and $s=1,3,6,8,10$ we check by calculation that

$$
f_{s}(x) \geqslant f_{s}\left(x_{\min }\right)=\left(\left(F(m) / m^{\theta}\right)^{1 /(1-\theta)}+\left(\gamma 2^{t}\right)^{1 /(1-\theta)}\right)^{1-\theta}>\gamma
$$

is fulfilled. For $m=n_{s}-1$ and $s=2,4,5,7,9,10$ we ascertain that in these cases $x_{\min }>2^{r-s}$. Then for $s \neq 10$,

$$
f_{s}(x) \geqslant f_{s}\left(2^{r-s}\right)=\frac{F\left(n_{s}-1\right)+\gamma 2^{t_{s}-1}}{n_{s}^{\theta}}=\frac{F\left(n_{s}\right)-(1-\gamma) 2^{t_{s}-1}}{n_{s}^{\theta}}>\gamma
$$

is seen to be true by calculation. In the case $m=n_{10}-1, s=10$, we first have

$$
f_{10}(x) \geqslant f_{10}\left(2^{r-11}\right)=\frac{3 F\left(n_{10}\right)-(3-\gamma) 2^{t_{10}-1}}{\left(2 n_{10}-1\right)^{\theta}}>\gamma, \quad 1 \leqslant x \leqslant 2^{r-11} .
$$

For the remaining partial interval

$$
n=2^{r-10}\left(n_{10}-1\right)+2^{r-11}+x=2^{r-11}\left(2 n_{10}-1\right)+x, \quad 1 \leqslant x \leqslant 2^{r-11} \text {, }
$$

we choose $m=2 n_{10}-1$ and $s=11$ in (12), and check the validity of (13).

Now the induction on $r$ is complete, and we have proved (11) for all $n$. Inequalities (10) and (11) then yield Theorem 2.

At the end we remark that $q$ from (10) probably will be the exact value of $\beta$. Moreover, we conjecture for all $r$,

$$
F(n) / n^{\theta} \geqslant q_{r} \text { for } 2^{r} \leqslant n \leqslant 2^{r+1} .
$$

It seems, however, that for a general proof we should know some more properties of the sequence of plus and minus signs beginning with (7). Are there any regularities in this sequence?

\section{REFERENCES}

1. N. J. Fine, Binomial coefficients modulo a prime, Amer. Math. Monthly 54 (1947), 589-592. MR 9, 331.

2. H. Harborth, Über die Teilbarkeit im Pascal-Dreieck, Math.-Phys. Semesterber. 22 (1975), 13-21.

3. D. Singmaster, Notes on binomial coefficients. III: Any integer divides almost all binomial coefficients, J. London Math. Soc. (2) 8 (1974), 555-560.

4. K. B. Stolarsky, Digital sums and binomial coefficients, Notices Amer. Math. Soc. 22 (1975), A-669. Abstract \#728-A7.

5. __ Power and exponential sums of digital sums related to binomial coefficient parity, SIAM J. Appl. Math. (to appear).

INSTITUT B FÜR MATHEMATIK, TECHNISCHE UNIVERSITAT BRAUNSCHWEIG, D3300 BRAUNSCHWEIG, WEST GERMANY 